论 文

\title{
基于碳酸钙-金纳米粒子无机杂化复合物固定硫堇 的新方法及其在过氧化氢传感器中的应用
}

\author{
李峰 ${ }^{(1)}$, 冯艳 ${ }^{(1)}$, 李晶晶 ${ }^{(1)}$, 郭杰 ${ }^{(2 *}$ \\ (1) 生态化工教育部重点实验室; 青岛科技大学化学与分子工程学院，青岛 266042 \\ (2) 吉林大学第二医院放疗科, 长春 130025 \\ *通讯作者, E-mail: lifeng@qust.edu.cn; guojie@jlu.edu.cn
}

收稿日期: 2010-05-11; 接收日期: 2010-06-24; 网络版发布日期: 2010-09-14 doi: 10.1007/s11426-010-4092-y

\begin{abstract}
摘要通过静电作用, 经碳酸钙-金纳米粒子 $\left(\mathrm{CaCO}_{3}-\mathrm{AuNPs}\right)$ 无机杂化复合物实现了电活性 物质硫堇(Th)在金电极表面的有效固定. AuNPs 静电吸附到 $\mathrm{CaCO}_{3}$ 微球表面形成 $\mathrm{CaCO}_{3}-\mathrm{AuNPs}$ 无机杂化复合物, 该杂化复合物具有微孔结构、大的表面积和好的生物相容性, 使得 Th 的固定 量和稳定性大大提高. 探讨了 Th 修饰电极在过氧化氢 $\left(\mathrm{H}_{2} \mathrm{O}_{2}\right)$ 传感器中的应用, 该传感器对 $\mathrm{H}_{2} \mathrm{O}_{2}$ 的响应快速, 线性范围宽 $\left(8.0 \times 10^{-7} \sim 1.06 \times 10^{-3} \mathrm{~mol} / \mathrm{L}\right)$, 检测限为 $2.0 \times 10^{-7} \mathrm{~mol} / \mathrm{L}$.

关键词

硫堇

$\mathrm{CaCO}_{3}-\mathrm{AuNPs}$

传感器

静电作用
\end{abstract}

\section{1 引言}

由于氧化还原酶的电活性中心包埋于其绝缘蛋 白质外壳内部, 因此, 实现酶的直接电化学通常比较 困难. 为此, 研究者使用碳纳米管 ${ }^{[1]}$ 、碳纳米角 ${ }^{[2]}$ 、纳 米粒子 ${ }^{[3]}$ 、导电聚合物 ${ }^{[4]}$ 和离子液体 ${ }^{[5]}$ 等物质实现酶 的直接电化学. 然而电子媒介体具有优异的电子传 递能力, 很容易在酶的氧化还原中心和电极之间建 立联系. 与无媒介体的生物传感器相比, 媒介体电化 学传感器还具有灵敏度高、检测限低、工作电位低和 抗干扰能力强等优点, 所以研究者对媒介体传感器 给予了极大的关注. 一些被广泛应用的电子媒介体 如铁氰酸盐 ${ }^{[6]}$ 、二茂铁及其衍生物 ${ }^{[7]}$ 、四硫富瓦烯 ${ }^{[8]}$ 、 奎宁-邻苯二亚胺 ${ }^{[9]}$ 、荎酚蓝 ${ }^{[10]}$ 、亚甲基绿 ${ }^{[11]}$ 和硫堇 $(T h)^{[12]}$ 等可直接加入到溶液中, 也可固定到电极表 面. 直接加入到溶液中, 存在媒介体污染样品、辅助 电极和参比电极的问题, 所以研究者提出了各种将 媒介体固定到支持基质的策略.

金纳米粒子(AuNPs)由于其好的生物相容性、纳 米尺寸效应和强的导电能力被广泛用来结合媒介体
构筑传感器 ${ }^{[13 ~ 15]}$. 在实际操作中, AuNPs 通常与其他 生物相容性的物质共同用于酶或其他生物分子的负 载, 如聚合物基质、溶胶-凝胶基质、无机微米/纳米 材料等, 它们之间的协同作用可以显著改善传感器 的性能. 其中的碳酸钙 $\left(\mathrm{CaCO}_{3}\right)$ 微球具有多孔的表 面、好的生物相容性、高的机械强度和大的表面积, 是制备无机杂化材料的良好选择 ${ }^{[16,17]}$. pH 7.0 条件下, 带正电的 $\mathrm{CaCO}_{3}$ 可静电吸引带负电的 AuNPs 形成核壳 $\mathrm{CaCO}_{3}-\mathrm{AuNPs}$ 无机杂化复合物, 该复合物兼具 $\mathrm{CaCO}_{3}$ 和 AuNPs 的良好性能, 在传感界面的构筑方 面具有很大的应用前景.

$\mathrm{Th}$ 是一种水溶性的染料, 具有电活性和光活性, 在近年的研究中受到广泛关注. 据此, 研究者们制备 了很多电化学传感器和光化学传感器 ${ }^{[18 ~ 20]}$. Th 通常 与酶共固定构筑生物传感器 ${ }^{[21 ~ 23]}$, 酶需要友好的环 境来保持自身的活性, 且构筑酶修饰电极成本较高. 而 $\mathrm{Th}$ 自身能在被分析物和电极之间传递电子, 制备 无酶型的 Th 修饰电极可避免酶电极自身存在的不稳 定和成本高的问题, 因此 $\mathrm{Th}$ 的固定方法成为研究者 
关注的焦点. 在众多 Th 作为媒介体的传感器中, 通 常采用的方法有吸附 ${ }^{[24]}$ 、溶胶-凝胶法 ${ }^{[25]}$ 、静电相互 作用 ${ }^{[26]}$ 、包覆 ${ }^{[27]}$ 、共价固定 ${ }^{[28,29]}$ 、电聚合 ${ }^{[30]}$ 和非电 化学沉积 ${ }^{[31]}$ 等. 其中, 静电作用由于其内在的简单性 受到人们的青睐.

本文通过静电作用将 Th 固定到 $\mathrm{CaCO}_{3}-\mathrm{AuNPs}$ 无机杂化复合物表面, 提出了一种简单可靠的固 定电活性物质 Th 的新方法. 探讨了 $\mathrm{Th}$ 修饰电极在 过氧化氢 $\left(\mathrm{H}_{2} \mathrm{O}_{2}\right)$ 传感器中的应用. 结果表明, 制备 的 $\mathrm{CaCO}_{3}-\mathrm{AuNPs}$ 复合物保留了 $\mathrm{CaCO}_{3}$ 的多孔结构, 可为 $\mathrm{Th}$ 的负载提供大的表面积. 并且, 该复合物具 有良好的导电性和生物相容性. 因此, 与未加 $\mathrm{CaCO}_{3}$ 的传感器相比, 所构筑的传感器对 $\mathrm{H}_{2} \mathrm{O}_{2}$ 的检 测显示出更高的灵敏度、更宽的线性范围和更低的检 测限.

\section{2 实验部分}

\section{1 仪器与试剂}

仪器: CHI 832B 电化学分析仪与 CHI 660C 电化 学分析仪 (上海辰华仪器公司); $\mathrm{Au}$ 电极及其修饰电 极为工作电极, 铂丝电极为对电极, $\mathrm{Ag} / \mathrm{AgCl}$ (饱和 $\mathrm{KCl}$ ) 电极为参比电极; SIRION 扫描电子显微镜(美 国 FEI).

试剂: $\mathrm{Th}$ 购自中国上海国药公司; 4-氨基苯硫酚 (ATP)购自 Aldrich; 各个浓度的 $\mathrm{H}_{2} \mathrm{O}_{2}$ 溶液用 $30 \%$ 的 $\mathrm{H}_{2} \mathrm{O}_{2}$ (中国南京化学试剂公司)稀释, 并用 $\mathrm{KMnO}_{4}$ 标 定. 氯化钻 $\left(\mathrm{CaCl}_{2}\right)$ 和碳酸钠 $\left(\left(\mathrm{Na}_{2} \mathrm{CO}_{3}\right)\right.$ 购自中国天津 博迪化学试剂公司; $\mathrm{HAuCl}_{4} \cdot 4 \mathrm{H}_{2} \mathrm{O}$ 购自 Sigma; 含 0.1 $\mathrm{mol} / \mathrm{L} \mathrm{KCl}$ 的不同 $\mathrm{pH}$ 值的 $0.1 \mathrm{~mol} / \mathrm{L}$ 磷酸盐缓冲液 (PBS)用 $0.1 \mathrm{~mol} / \mathrm{L} \mathrm{NaH} \mathrm{PO}_{4}$ 和 $\mathrm{Na}_{2} \mathrm{HPO}_{4}$ 溶液配制, 并用 $0.1 \mathrm{~mol} / \mathrm{L} \mathrm{H}_{3} \mathrm{PO}_{4}$ 或 $\mathrm{NaOH}$ 调节 $\mathrm{pH}$ 值; 实验用 水为二次蒸馏水 $(\mathrm{DDW})$.

\section{$2.2 A u N P s$ 和 $\mathrm{CaCO}_{3}$ 微球的制备}

实验中用到的玻璃仪器首先在新鲜制备的 $\mathrm{HNO}_{3}-\mathrm{HCl}(3: 1, V / V)$ 溶液中清洗, 用 DDW 充分洗涤 后, 在空气中干燥. AuNPs 采用经典的柠檬酸盐在水 溶液中还原 $\mathrm{HAuCl}_{4}$ 的方法制备. 简单来说: 将 10 $\mathrm{mL} 38.8 \mathrm{mmol} / \mathrm{L}$ 柠檬酸钠溶液加入到 $100 \mathrm{~mL} 1.0$ $\mathrm{mmol} / \mathrm{L}$ 沸腾的 $\mathrm{HAuCl}_{4}$ 溶液中, 当混合液颜色变为酒
红色时即有 AuNPs 生成. 通过透射电子显微镜表征, 制得 AuNPs 的尺寸为 $13 \mathrm{~nm}$.

根据 $\mathrm{Cai}$ 等 ${ }^{[32]}$ 提出的方法制备 $\mathrm{CaCO}_{3}$ 微球. 室温 条件下, $0.33 \mathrm{~mol} / \mathrm{L} \mathrm{Na}_{2} \mathrm{CO}_{3}$ 溶液加入到等体积的 $0.33 \mathrm{~mol} / \mathrm{L} \mathrm{CaCl}$ 溶液中, 摚拌 $1 \mathrm{~min}$. 将沉淀的 $\mathrm{CaCO}_{3}$ 离心分离, 用 $\mathrm{DDW}$ 冲洗三次后干燥.

\section{$2.3 \mathrm{CaCO}_{3}-\mathrm{AuNPs}$ 无机杂化复合物的制备}

$10 \mathrm{mg} \mathrm{CaCO}_{3}$ 加入到 $10 \mathrm{~mL}$ 金胶溶液( $\mathrm{pH}$ 7.0)中 超声 $5 \mathrm{~min}$ 使 $\mathrm{CaCO}_{3}$ 分散. 离心 $2 \mathrm{~min}$ 后, 得到浅紫 色的 $\mathrm{CaCO}_{3}-\mathrm{AuNPs}$ 无机杂化复合物. 用 DDW 洗涤 该复合物后干燥 ${ }^{[32]}$.

\section{$2.4 \mathrm{Th}$ 经 $\mathrm{CaCO}_{3}-\mathrm{AuNPs}$ 无机杂化复合物固定到 电极表面}

修饰之前, 将裸 $\mathrm{Au}$ 电极在 $30 \% \mathrm{H}_{2} \mathrm{O}_{2}$ 与浓 $\mathrm{H}_{2} \mathrm{SO}_{4}$ 的混合液 $(3: 7, V / V)$ 中浸泡 $30 \mathrm{~min}$, 依次用 $1.0 、 0.3$ 和 $0.05 \mu \mathrm{m} \alpha-\mathrm{Al}_{2} \mathrm{O}_{3}$ 抛光后, 分别在 $\mathrm{DDW}$ 与乙醇溶液中 超声清洗, 然后在室温下干燥.

将抛光后的裸 $\mathrm{Au}$ 电极在 $5 \mathrm{mmol} / \mathrm{L}$ ATP 的乙醇 溶液中温育 $12 \mathrm{~h}$ 得到 ATP 单分子层修饰的 Au 电极, 然后分别用乙醇和 DDW 充分洗涤修饰电极除去未组 装的 ATP 分子. 将 $10 \mu \mathrm{L} 5 \mathrm{mg} / \mathrm{mL} \mathrm{CaCO}_{3}-\mathrm{AuNPs}$ 复 合物悬浮液滴涂到 ATP 修饰的 Au 电极表面保持 $3 \mathrm{~h}$ 后, 用 $0.1 \mathrm{~mol} / \mathrm{L} \mathrm{PBS}(\mathrm{pH} 7.0)$ 清洗, 得到 $\mathrm{CaCO}_{3}-$ AuNPs/ATP/Au 电极. 将上述修饰电极浸入 $2 \mathrm{mmol} / \mathrm{L}$ $\mathrm{Th}$ 溶液中 $6 \mathrm{~h}$, 使 $\mathrm{Th}$ 固定到 $\mathrm{CaCO}_{3}-\mathrm{AuNPs}$ 修饰电极 表面, 用 $0.1 \mathrm{~mol} / \mathrm{L} \mathrm{PBS}(\mathrm{pH}$ 7.0)清洗, 即制得 $\mathrm{Th} /$ $\mathrm{CaCO}_{3}-\mathrm{AuNPs} / \mathrm{ATP} / \mathrm{Au}$ 电极. 为了比较电极性能, 采 用同样的方法制备不含 $\mathrm{CaCO}_{3}$ 的 $\mathrm{Th} / \mathrm{AuNPs} / \mathrm{ATP} / \mathrm{Au}$ 电极. 各修饰电极不使用时于 $4{ }^{\circ} \mathrm{C}$ 下放置保存.

\section{5 电化学测定}

将各修饰电极置于 PBS 溶液中, 用 CHI 832B 电 化学分析仪记录循环伏安曲线, 扫描速率为 $50 \mathrm{mV} / \mathrm{s}$; 测定时间-电流曲线时, 应用电压设为 $-0.25 \mathrm{~V}$, 用 CHI 832B 电化学分析仪记录该传感器对连续滴加不 同浓度的 $\mathrm{H}_{2} \mathrm{O}_{2}$ 的响应. 在含 $0.1 \mathrm{~mol} / \mathrm{L} \mathrm{KCl}$ 的 1.0 $\mathrm{mmol} / \mathrm{L} \mathrm{K} \mathrm{K}_{3} \mathrm{Fe}(\mathrm{CN})_{6} / \mathrm{K}_{4} \mathrm{Fe}(\mathrm{CN})_{6}(1: 1)$ 溶液中, 用 $\mathrm{CHI}$ 660C 电化学分析仪记录各修饰电极的电化学交流阻 抗(EIS) 曲线, 应用电压 $5 \mathrm{mV}$, 外加电位为 $0.221 \mathrm{~V}$, 频率范围为 $10^{4} \sim 10^{-1} \mathrm{~Hz}$. 


\section{3 结果与讨论}

\section{$3.1 \mathrm{Th}$ 经 $\mathrm{CaCO}_{3}-\mathrm{AuNPs}$ 无机杂化复合物固定到} 电极表面

电活性物质 $\mathrm{Th}$ 分子通过静电作用经 $\mathrm{CaCO}_{3}-$ AuNPs 无机杂化复合物固定到 $\mathrm{Au}$ 电极表面. 如图 1 所示, $\mathrm{CaCO}_{3}$ 的等电点为 $8.5, \mathrm{pH}$ 为 7.0 时, $\mathrm{CaCO}_{3}$ 微 球带正电 ${ }^{[33]}$. 在相同 $\mathrm{pH}$ 条件下, AuNPs 带负电. AuNPs 即可通过静电作用吸附到 $\mathrm{CaCO}_{3}$ 微球表面形 成 $\mathrm{CaCO}_{3}$-AuNPs 无机杂化复合物. AuNPs 吸附到 $\mathrm{CaCO}_{3}$ 微球表面可避免 $\mathrm{CaCO}_{3}$ 从多孔的六方晶系球 文石转化为三方晶系方解石. 同时, $\mathrm{CaCO}_{3}$ 也为 AuNPs 提供机械支撑, 使 AuNPs 免受损失. 因此, 形 成的 $\mathrm{CaCO}_{3}-\mathrm{AuNPs}$ 无机杂化复合物具有良好的稳定 性 ${ }^{[32]}$. ATP 可通过 $\mathrm{Au}-\mathrm{S}$ 键自组装在 $\mathrm{Au}$ 电极表面形 成氨基修饰的电极表面 ${ }^{[34]}$, 外层氨基即可通过 $\mathrm{Au}-\mathrm{NH}_{2}$ 相互作用使 $\mathrm{CaCO}_{3}-\mathrm{AuNPs}$ 无机杂化复合物 固定到电极表面 ${ }^{[35]}$. pH 7.0 条件下, AuNPs 表面带负 电荷, 这样 $\mathrm{CaCO}_{3}-\mathrm{AuNPs}$ 复合物即可吸附带正电的 Th. AuNPs 一方面被 $\mathrm{CaCO}_{3}$ 静电吸引, 另一方面被 Th 所带的正电荷及其氨基所稳定, 所以 $\mathrm{CaCO}_{3} 、 \mathrm{AuNPs}$ 和 Th 共同形成稳定的“三明治夹心”结构, 提高了固 定到电极表面的 Th 的稳定性.

\section{2 $\mathrm{CaCO}_{3}-\mathrm{AuNPs}$ 无机杂化复合物的表征}

扫描电子显微镜 (SEM) 是表征不同物质表面形 貌的有效手段, 图 2 显示了 $\mathrm{CaCO}_{3}$ 微球吸附 AuNPs 前(a)后(b)的图像. 显然, 制备的 $\mathrm{CaCO}_{3}$ 微球具有多

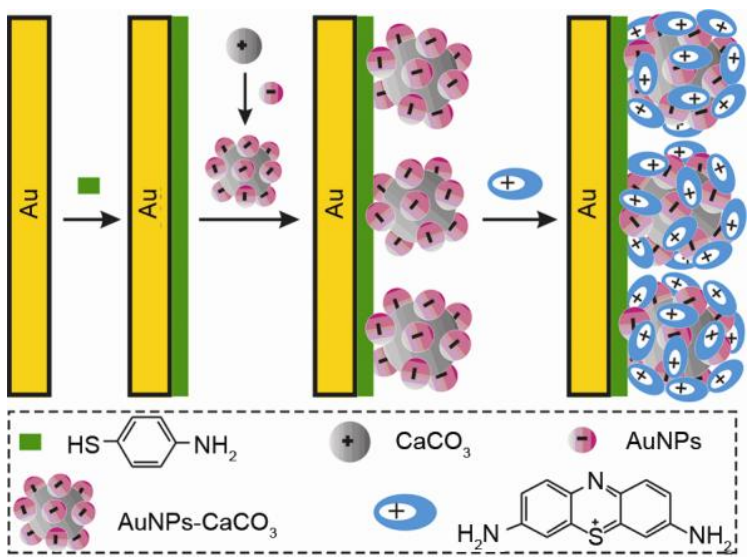

图 $1 \mathrm{Th}$ 经 $\mathrm{CaCO}_{3}-\mathrm{AuNPs}$ 无机杂化复合物固定到 $\mathrm{Au}$ 电极 表面的示意图

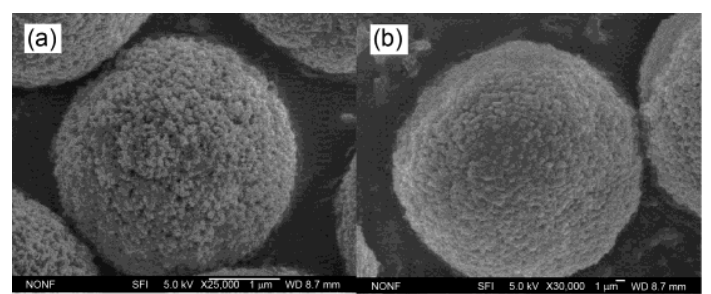

图 $2 \mathrm{CaCO}_{3}$ 微球吸附 AuNPs 前(a)后(b)的扫描电镜图

孔表面, 可为 AuNPs 的吸附提供大的表面积. 得到 的 $\mathrm{CaCO}_{3}-\mathrm{AuNPs}$ 无机杂化复合物保留了 $\mathrm{CaCO}_{3}$ 微球 的多孔结构, 并且微球表面变得相对光滑. AuNPs 均 匀地分布在 $\mathrm{CaCO}_{3}$ 微球的孔中, 表明 AuNPs 成功吸 附到 $\mathrm{CaCO}_{3}$ 微球表面.

\section{3 传感器的电化学表征}

图 3 显示了不同修饰电极在 $0.1 \mathrm{~mol} / \mathrm{L}$ PBS $(\mathrm{pH}$ 6.0)中, 扫描速率为 $50 \mathrm{mV} / \mathrm{s}$ 的循环伏安图. 在裸 $\mathrm{Au}$ 电极 (曲线 a)、 $\mathrm{ATP} / \mathrm{Au}$ (曲线 b) 和 $\mathrm{CaCO}_{3}-\mathrm{AuNPs}$ / $\mathrm{ATP} / \mathrm{Au}$ (曲线 $\mathrm{c}$ ) 上都没有氧化还原峰出现, 而 $\mathrm{Th} / \mathrm{CaCO}_{3}-\mathrm{AuNPs} / \mathrm{ATP} / \mathrm{Au}$ (曲线 e) 出现了一对可逆性 好的峰(相对于 $\mathrm{Ag} / \mathrm{AgCl}$ 电极, 标准电位为 $-0.19 \mathrm{~V}$ ), 氧化还原峰电位分别为 -0.15 和 $-0.23 \mathrm{~V}$. Th/AuNPs/ $\mathrm{ATP} / \mathrm{Au}$ (曲线 d)也出现了一对 $\mathrm{Th}$ 的峰, 但与 $\mathrm{Th} /$ $\mathrm{CaCO}_{3}-\mathrm{AuNP}$ s/ATP/Au 相比, 峰电位未有明显移动, 但其峰电流明显减小. 根据法拉第定律 $Q=n F A \Gamma$, 其中 $A$ 为电极的实际面积 $\left(\mathrm{cm}^{2}\right), \Gamma$ 为电极表面电活性

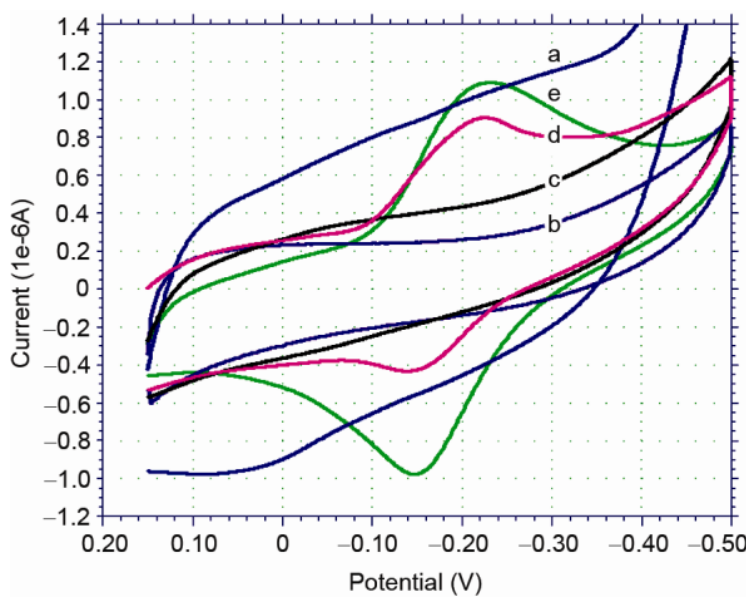

图 3 裸 $\mathrm{Au}$ 电极(a), $\mathrm{ATP} / \mathrm{Au}(\mathrm{b}), \mathrm{CaCO}_{3}-\mathrm{AuNPs} / \mathrm{ATP} / \mathrm{Au}$ (c), $\mathrm{Th} / \mathrm{AuNPs} / \mathrm{ATP} / \mathrm{Au}(\mathrm{d})$ 和 $\mathrm{Th} / \mathrm{CaCO}_{3}-\mathrm{AuNPs} / \mathrm{ATP} / \mathrm{Au}$ (e) 在 $0.1 \mathrm{~mol} / \mathrm{L} \mathrm{PBS}(\mathrm{pH}$ 6.0)中, 扫描速率为 $50 \mathrm{mV} / \mathrm{s}$ 的循环伏 安图 
物质的覆盖率 $\left(\mathrm{mol} / \mathrm{cm}^{2}\right), n$ 为电子转移数, $F$ 为法拉第 常数 $(96485 \mathrm{C} / \mathrm{mol})$. 得到 $\mathrm{Th} / \mathrm{CaCO}_{3}-\mathrm{AuNPs} / \mathrm{ATP} / \mathrm{Au}$ 电极表面 $\mathrm{Th}$ 的表面覆盖率为 $5.80 \times 10^{-10} \mathrm{~mol} / \mathrm{cm}^{2}$, 而 Th/AuNPs/ATP/Au 电极表面的 $\mathrm{Th}$ 的表面覆盖率为 $4.17 \times 10^{-10} \mathrm{~mol} / \mathrm{cm}^{2}$, 表明多孔 $\mathrm{CaCO}_{3}$ 的存在可显著 提高 $\mathrm{Th}$ 的负载量. 这可能是由于 $\mathrm{CaCO}_{3}$ 的多孔结构 为 AuNPs 的吸附提供了三维空间, 增大了 $\mathrm{Th}$ 的固定 量. 同时 $\mathrm{CaCO}_{3}-\mathrm{AuNPs}$ 无机杂化复合物良好的导电 性, 有效地促进了 $\mathrm{Th}$ 分子与电极表面可逆的电子传 递.

研究了扫描速率对 $\mathrm{Th} / \mathrm{CaCO}_{3}-\mathrm{AuNPs} / \mathrm{ATP} / \mathrm{Au}$ 电 极表面固定的 Th 的电化学响应的影响. 不同扫速下 得到的循环伏安曲线都具有对称的氧化还原峰, 显 示出可逆的电极反应过程. 随着扫描速率的增加, 氧 化峰电位 $\left(E_{\mathrm{pa}}\right)$ 和还原峰电位 $\left(E_{\mathrm{pc}}\right)$ 分别向正电位和负 电位方向移动, 氧化峰电流和还原峰电流与扫描速 率在 $20 \sim 400 \mathrm{mV} / \mathrm{s}$ 范围内呈正比, 表明电极反应过 程为吸附控制过程. 当 $n \Delta E_{\mathrm{p}}<200 \mathrm{mV}$ 时, $E_{\mathrm{pc}}$ 和 $E_{\mathrm{pa}}$ 分别与扫描速率 $(v)$ 的对数呈正比, 线性回归方程分 别为 $E_{\mathrm{pa}}=-0.11825+0.01064 \ln v$ 和 $E_{\mathrm{pc}}=-0.25821+$ $0.00848 \ln v$. 根据 Laviron 理论 ${ }^{[36]}: E_{\mathrm{pa}}=\mathrm{A}+(R T$ $/(1-\alpha) n A F) \ln v$ 和 $E_{\mathrm{pc}}=\mathrm{B}-(R T / \alpha n A F) \ln v$, 得到电子转 移系数 $\alpha$ 为 0.55 , 该值接近理论值 0.5 , 符合准可逆过 程的特征. 再根据公式:

$$
\begin{aligned}
\log K_{\mathrm{s}}= & \alpha \log (1-\alpha)+(1-\alpha) \log \alpha-\log (R T / n F v) \\
& -\alpha(1-\alpha) n F \Delta E \mathrm{p} / 2.3 R T
\end{aligned}
$$

得到扫描速率为 $50 \mathrm{mV} / \mathrm{s}$ 时, 氧化还原过程电子转移 速率常数 $K_{\mathrm{s}}$ 为 $0.398 \mathrm{~s}^{-1}$, 表明高导电性的 $\mathrm{CaCO}_{3}-\mathrm{AuNPs}$ 无机杂化复合物可有效地促进 Th 与 电极表面的电子转移.

为考察 $\mathrm{Th} / \mathrm{CaCO}_{3}-\mathrm{AuNPs} / \mathrm{ATP} / \mathrm{Au}$ 在分析中的实 际应用, 研究了该修饰电极对 $\mathrm{H}_{2} \mathrm{O}_{2}$ 的催化性能. 图 4 显示了 $\mathrm{Th} / \mathrm{CaCO}_{3}-\mathrm{AuNPs} / \mathrm{ATP} / \mathrm{Au}$ 在有无 $\mathrm{H}_{2} \mathrm{O}_{2}$ 存在 的情况下得到的循环伏安图. 当 $80 \mu \mathrm{mol} / \mathrm{L} \mathrm{H}_{2} \mathrm{O}_{2}$ 加入 到 $0.1 \mathrm{~mol} / \mathrm{L}$ PBS ( $\mathrm{pH}$ 6.0)中时(曲线(b)), 还原峰电流 增大, 氧化峰电流减小, 是典型的 $\mathrm{Th}$ 对 $\mathrm{H}_{2} \mathrm{O}_{2}$ 的催化 行为. 其反应机理如下:

Thionine(Red) $+\mathrm{H}_{2} \mathrm{O}_{2} \stackrel{\mathrm{pH} 6.0}{\longrightarrow}$ Thionine $(\mathrm{Ox})+\mathrm{H}_{2} \mathrm{O}$

Thionine(Ox) $+2 \mathrm{e}^{-}+\mathrm{H}^{+} \stackrel{\mathrm{pH} 6.0}{\longrightarrow}$ Thionine(Red)

EIS 可提供每一步修饰过程电极表面的阻抗变 化情况. 图 5 显示了裸 $\mathrm{Au}$ 电极(a), ATP/Au(b),

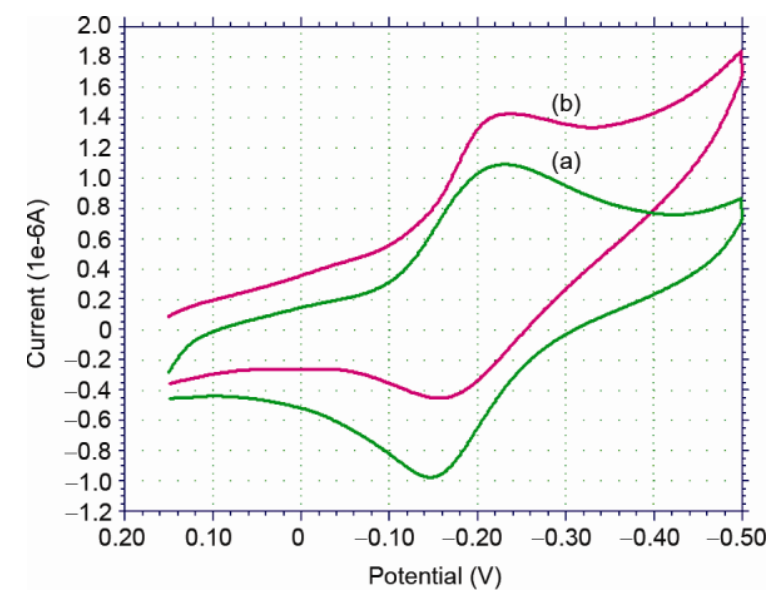

图 $4 \mathrm{Th} / \mathrm{CaCO}_{3}-\mathrm{AuNPs} / \mathrm{ATP} / \mathrm{Au}$ 在 $0.1 \mathrm{~mol} / \mathrm{L} \mathrm{PBS}(\mathrm{pH}$ 6.0) 中未加入 $\mathrm{H}_{2} \mathrm{O}_{2}$ (a) 和加入 $80 \mu \mathrm{mol} / \mathrm{L} \mathrm{H}_{2} \mathrm{O}_{2}$ (b), 扫描速率为 $50 \mathrm{mV} / \mathrm{s}$ 的循环伏安图

$\mathrm{CaCO}_{3}$-AuNPs/ATP/Au(c)和 $\mathrm{Th} / \mathrm{CaCO}_{3}$-AuNPs/ATP/ $\mathrm{Au}(\mathrm{d})$ 在含 $0.1 \mathrm{~mol} / \mathrm{L} \mathrm{KCl}$ 的 $1.0 \mathrm{mmol} / \mathrm{L} \mathrm{K}_{3} \mathrm{Fe}(\mathrm{CN})_{6}$ $/ \mathrm{K}_{4} \mathrm{Fe}(\mathrm{CN})_{6}(1: 1)$ 溶液中测得的交流阻抗谱图. 从图 中可以看出, ATP 自组装到裸 Au 电极表面后(曲线 b), 其电子传递阻抗 $\left(R_{\mathrm{et}}\right)$ 与裸 $\mathrm{Au}$ 电极(曲线 a)相比明显 增大到 $2950 \Omega$, 表明 ATP 单分子层阻碍了电子的传 递. 与之相反, 当 $\mathrm{CaCO}_{3}-\mathrm{AuNPs}$ 无机杂化复合物固 定到 ATP 修饰电极表面时(曲线 $\mathrm{c}$ ), $R_{\mathrm{et}}$ 值减小为 1834 $\Omega$, 表明 $\mathrm{CaCO}_{3}-\mathrm{AuNPs}$ 复合物可促进电子转移. 如 我们所料, $\mathrm{Th} / \mathrm{CaCO}_{3}-\mathrm{AuNPs} / \mathrm{ATP} / \mathrm{Au}$ 的 $R_{\mathrm{et}}$ 值与

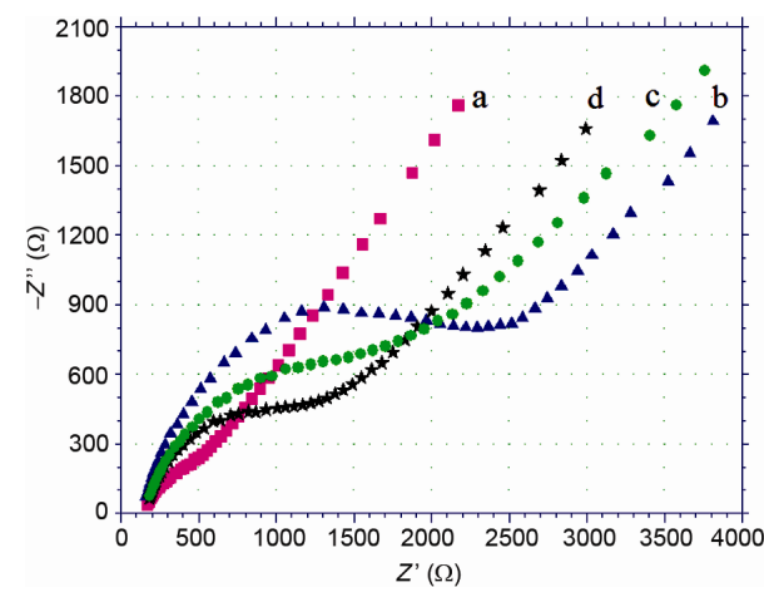

图 5 不同修饰电极在含 $0.1 \mathrm{~mol} / \mathrm{L} \mathrm{KCl}$ 的 $1.0 \mathrm{mmol} / \mathrm{L}$ $\mathrm{K}_{3} \mathrm{Fe}(\mathrm{CN})_{6} / \mathrm{K}_{4} \mathrm{Fe}(\mathrm{CN})_{6}(1: 1)$ 溶液中的交流阻抗谱图. (a) 裸 $\mathrm{Au}$ 电极, (b) ATP/Au, (c) $\mathrm{CaCO}_{3}-\mathrm{AuNPs} / \mathrm{ATP} / \mathrm{Au}$ 和 (d) $\mathrm{Th} / \mathrm{CaCO}_{3}-\mathrm{AuNPs} / \mathrm{ATP} / \mathrm{Au}$ 
$\mathrm{CaCO}_{3}-\mathrm{AuNPs} / \mathrm{ATP} / \mathrm{Au}$ 的相比显著减小, 这归因于 带正电的 $\mathrm{Th}$ 分子与带负电的 $\mathrm{Fe}(\mathrm{CN})_{6}{ }^{3-14-}$ 间强的吸引 作用, 同时表明 $\mathrm{Th}$ 成功固定到 $\mathrm{CaCO}_{3}-\mathrm{AuNPs}$ 复合物 表面.

\section{4 溶液 pH 值对传感器性能的影响}

在不同 $\mathrm{pH}$ 值(3.0 7.0)的 PBS 中, 对 $\mathrm{Th} / \mathrm{CaCO}_{3}-$ AuNPs/ATP/Au 电极的循环伏安行为进行了研究. 随 着 $\mathrm{pH}$ 值的增大, $E_{\mathrm{pa}}$ 和 $E_{\mathrm{pc}}$ 均向负电位方向移动, 但是 电势差只有轻微改变, 表明固定的 $\mathrm{Th}$ 在电极反应中 具有良好的可逆性. 同时, 传感器的电流响应逐渐减 小. 在酸性条件下, 该传感器显示出较大的峰电流和 对称的氧化还原峰, 但是 $\mathrm{CaCO}_{3}$ 会有部分溶解, 这将 会对传感器的灵敏度和稳定性产生重大影响. 因此, 本文选择 $\mathrm{pH} 6.0$ 进行实验测定.

\section{5 传感器对不同浓度 $\mathrm{H}_{2} \mathrm{O}_{2}$ 的电流响应}

图 6 显示了 $\mathrm{Th} / \mathrm{CaCO}_{3}-\mathrm{AuNPs} / \mathrm{ATP} / \mathrm{Au}(1)$ 和 $\mathrm{Th} /$

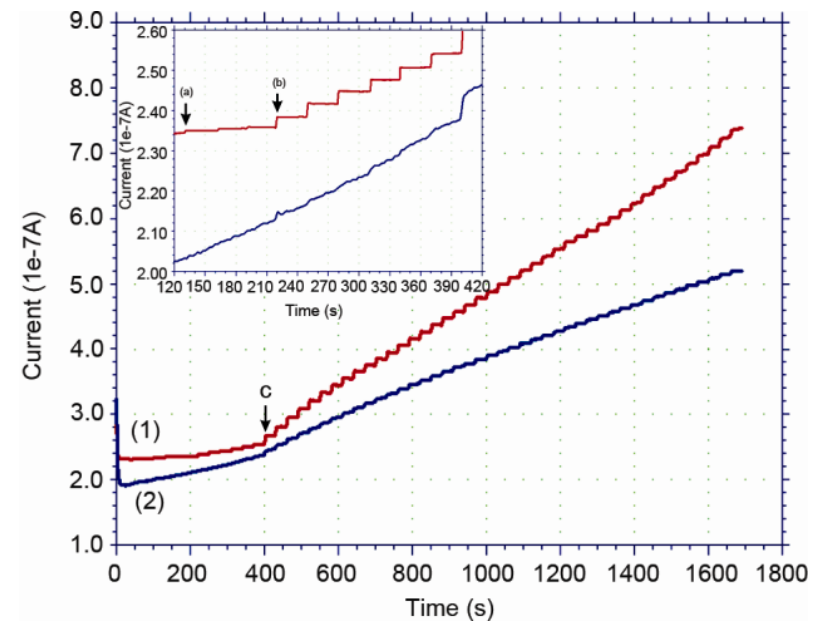

图 $6 \mathrm{Th} / \mathrm{CaCO}_{3}-\mathrm{AuNPs} / \mathrm{ATP} / \mathrm{Au}(1)$ 和 $\mathrm{Th} / \mathrm{AuNPs} / \mathrm{ATP} / \mathrm{Au}(2)$ 在 $0.1 \mathrm{~mol} / \mathrm{L}$ PBS (pH 6.0)中对不同浓度 $\mathrm{H}_{2} \mathrm{O}_{2}$ 响应的电流-时 间曲线. $\mathrm{H}_{2} \mathrm{O}_{2}$ 的浓度分别为: 0.8 (a), 4.0 (b) 和 $24 \mu \mathrm{mol} / \mathrm{L}$ (c); 内插图为前 $400 \mathrm{~s}$ 传感器对 $\mathrm{H}_{2} \mathrm{O}_{2}$ 的响应

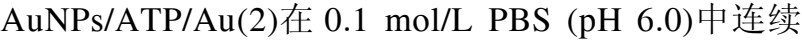
滴加不同浓度的 $\mathrm{H}_{2} \mathrm{O}_{2}$ 得到的电流-时间曲线. 摚拌 条件下, 加入一定量的 $\mathrm{H}_{2} \mathrm{O}_{2}$ 后, 两种传感器的还原 电流阶梯上升并达到稳定状态. 但 $\mathrm{Th} / \mathrm{CaCO}_{3}-\mathrm{AuNPs} /$ $\mathrm{ATP} / \mathrm{Au}$ 对 $\mathrm{H}_{2} \mathrm{O}_{2}$ 的响应更为迅速，达到 $95 \%$ 的稳 态电流小于 $3 \mathrm{~s}$, 并且响应也更为灵敏. 这表明由于 多孔 $\mathrm{CaCO}_{3}$-AuNPs 无机杂化复合物的存在使 $\mathrm{H}_{2} \mathrm{O}_{2}$ 向 $\mathrm{Th} / \mathrm{CaCO}_{3}-\mathrm{AuNPs} / \mathrm{ATP} / \mathrm{Au}$ 表面的扩散更为迅速. 在最优化的实验条件下, 该传感器测定 $\mathrm{H}_{2} \mathrm{O}_{2}$ 的线 性 回归方程为 $I\left(10^{-7} \mathrm{~A}\right)=0.00455 C\left(10^{-6} \mathrm{~mol} / \mathrm{L}\right)+$ 0.12477 , 其线性范围为 $8.0 \times 10^{-7} \sim 1.06 \times 10^{-3}$ $\mathrm{mol} / \mathrm{L}$, 相关系 数为 $0.9994(n=52)$, 检测限为 $2.0 \times$ $10^{-7} \mathrm{~mol} / \mathrm{L}$.

该传感器测定 $\mathrm{H}_{2} \mathrm{O}_{2}$ 的响应时间、线性范围、检 测限等特征量与其他传感器进行了比较, 如表 1 所 示. $\mathrm{Th} / \mathrm{CaCO}_{3}-\mathrm{AuNPs} / \mathrm{ATP} / \mathrm{Au}$ 修饰电极对 $\mathrm{H}_{2} \mathrm{O}_{2}$ 的电

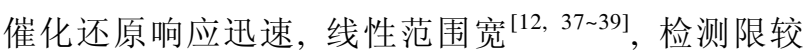
低 ${ }^{[12,39]}$.

根据 Lineweaver-Burk 方程 ${ }^{[40]}$, 其中 $\boldsymbol{I}_{\mathrm{ss}}$ 为加入底 物后的稳态电流, $I_{\max }$ 为最大电流, $K_{\mathrm{M}}^{\mathrm{app}}$ 为表观米氏 常数, $C$ 为底物浓度. 以 $1 / I_{\mathrm{ss}}$ 对 $1 / C$ 作图, 由截距可得 $I_{\max }$ ，再根据斜率计算得到 $K_{\mathrm{M}}^{\mathrm{app}}$ 为 $4.49 \times 10^{-5} \mathrm{~mol} / \mathrm{L}$, 表明 $\mathrm{Th}$ 对底物 $\mathrm{H}_{2} \mathrm{O}_{2}$ 具有较强的亲和性.

$$
\frac{1}{I_{\mathrm{ss}}}=\frac{1}{I_{\max }}+\frac{K_{\mathrm{M}}^{\mathrm{app}}}{I_{\max } C}
$$

\section{6 传感器的重现性和稳定性}

对 $50 \mu \mathrm{mol} / \mathrm{L} \mathrm{H}_{2} \mathrm{O}_{2}$ 进行 11 次平行测定的相对标 准偏差(RSD)为 $3.0 \%$. 在同样的操作条件下, 考察了 不同电极的重现性. 6 根独立制备的传感器对 50 $\mu \mathrm{mol} / \mathrm{L} \mathrm{H}_{2} \mathrm{O}_{2}$ 的响应的 RSD 为 $3.5 \%$. 传感器在 $4{ }^{\circ} \mathrm{C}$ 下保存 $30 \mathrm{~d}$ 后, 对 $50 \mu \mathrm{mol} / \mathrm{L} \mathrm{H}_{2} \mathrm{O}_{2}$ 的响应仍可达到原 始响应的 $97.9 \%$, 说明传感器具有良好的稳定性. 这 可能是由于 $\mathrm{CaCO}_{3}-\mathrm{AuNPs}$ 无机杂化复合物的多孔表

表 1 不同修饰电极测定 $\mathrm{H}_{2} \mathrm{O}_{2}$ 分析性能的比较

\begin{tabular}{ccccc}
\hline 修饰电极 & 响应时间 $(\mathrm{s})$ & 线性范围 $(\mu \mathrm{mol} / \mathrm{L})$ & 检测限 $(\mu \mathrm{mol} / \mathrm{L})$ & 文献 \\
\hline Th-NWs/GCE & $4 \sim 7$ & $5 \sim 2800$ & - & {$[12]$} \\
Th/MWCNT/Nf/PIGE & 4 & $1.37 \sim 344$ & 8.3 & {$[37]$} \\
$\mathrm{Hb}-\mathrm{CHT} / \mathrm{nano}-\mathrm{CaCO}_{3} / \mathrm{GCE}$ & 5 & $37 \sim 8300$ & 0.081 & {$[38]$} \\
$\mathrm{HRP} / \mathrm{CS} / \mathrm{GPTMS} / \mathrm{GCE}$ & 5 & $0.2 \sim 46$ & 0.2 & {$[39]$} \\
$\mathrm{Th} / \mathrm{CaCO}_{3}-\mathrm{AuNPs} / \mathrm{ATP} / \mathrm{Au}$ & 3 & $0.8 \sim 10600$ & proposed \\
\hline
\end{tabular}


面保护 Th 免受损失, 也为其提供了友好环境, 更好 地保持了 $\mathrm{Th}$ 的电活性.

\section{7 干扰实验和传感器对实际样品的测定}

在 $\mathrm{H}_{2} \mathrm{O}_{2}$ 的检测中, 抗坏血酸 $(A A)$ 、多巴胺(DA)、 尿酸 $(\mathrm{UA})$ 和葡萄糖(Glucose) 都是潜在的干扰. 本实 验中, $100 \mu \mathrm{mol} / \mathrm{L}$ AA、DA、UA 和 Glucose 对 50 $\mu \mathrm{mol} / \mathrm{L} \mathrm{H}_{2} \mathrm{O}_{2}$ 的测定引起的干扰均可以忽略, 这可能 是由于这些物质的氧化电位一般高于 $0 \mathrm{~V}$, 所以本实 验中- $0.25 \mathrm{~V}$ 的应用电位显著降低了干扰效应. 为评 价制备的传感器的实际应用性能, 对三份 $\mathrm{H}_{2} \mathrm{O}_{2}$ 的实 际样品进行了加标回收率的测定. 采用标准加入法, 实验结果(表 2) 显示该传感器对三份 $\mathrm{H}_{2} \mathrm{O}_{2}$ 实际样品的 加标回收率在 $97.2 \%$ 103.9\%之间, 表明该传感器可 用于实际样品的分析.
表 2 实际样品中 $\mathrm{H}_{2} \mathrm{O}_{2}$ 含量的测定

\begin{tabular}{cccc}
\hline$C_{\text {Original }}(\mu \mathrm{mol} / \mathrm{L})$ & $C_{\text {Added }}(\mu \mathrm{mol} / \mathrm{L})$ & $C_{\text {Found }}(\mu \mathrm{mol} / \mathrm{L})$ & Recovery $(\%)$ \\
\hline 20.00 & 45.00 & 63.72 & 97.2 \\
50.00 & 120.00 & 173.38 & 102.8 \\
100.00 & 200.00 & 307.83 & 103.9 \\
\hline
\end{tabular}

实验结果均为 3 次测定的平均值

\section{4 结论}

电活性物质 $\mathrm{Th}$ 成功固定到 $\mathrm{CaCO}_{3}-\mathrm{AuNPs}$ 无机 杂化复合物修饰的 $\mathrm{Au}$ 电极表面. 由于该复合物多孔 的结构、良好的生物相容性和强的导电能力, Th 的固 定量和稳定性得到提高. 所制备的传感器灵敏度高, 对 $\mathrm{H}_{2} \mathrm{O}_{2}$ 具有较强的催化还原能力. 建立的简便、可 靠、成本低的传感界面有望在其他传感器的研究中得 到发展.

\section{参考文献}

1 Yang J, Zhang RY, Xu Y, He PG, Fang YZ. Direct electrochemistry study of glucose oxidase on Pt nanoparticle-modified aligned carbon nanotubes electrode by the assistance of chitosan-CdS and its biosensoring for glucose. Electrochem Commun, 2008, 10: 1889-1892

2 Liu XQ, Shi LH, Niu WX, Li HJ, Xu GB. Amperometric glucose biosensor based on single-walled carbon nanohorns. Biosens Bioelectron, 2008, 23: 1887-1890

3 Li F, Feng Y, Wang Z, Yang LM, Zhuo LH, Tang B. Direct electrochemistry of horseradish peroxidase immobilized on the layered calcium carbonate-gold nanoparticals inorganic hybrid composite. Biosens Bioelectron, 2010, 25: 2244-2248

4 Komathia S, Gopalana AI, Lee KP. Fabrication of a novel layer-by-layer film based glucose biosensor with compact arrangement of multi-components and glucose oxidase. Biosens Bioelectron, 2009, 24: 3131-3134

5 Maleki N, Safavi A, Sedaghati F, Tajabadi F. Efficient electrocatalysis of L-cysteine oxidation at carbon ionic liquid electrode. Anal Biochem, 2007, 369: 149-153

6 Schubert F, Saini S, Turner APF. Mediated amperometric enzyme electrode incorporating peroxidase for the determination of hydrogen peroxide in organic solvents. Anal Chim Acta, 1991, 245: 133-138

7 Sanchez PD, Ordieres AJM, Garcia AC, Blanco PT. Peroxidase-ferrocene modified carbon paste electrode as an amperometric sensor for the hydrogen peroxide assay. Electroanalysis, 1991, 3:281-285

8 Wendzinski F, Grundig B, Renneberg R, Spener F. Highly sensitive determination of hydrogen peroxide and peroxidase with tetrathiafulvalene-based electrodes and the application in immunosensing. Biosens Bioelectron, 1997, 12: 43-52

9 Wang J, Frieha B, Naser N, Romero EG, Wollenberger U, Ozsoz M, Evans O. Amperometric biosensing of organic peroxides with peroxidase-modified electrodes. Anal Chim Acta, 1991, 254: 81-88

10 Liu H, Zhang X, Wei J. An amperometric meldola blue-mediated sensor high sensitive to hydrogen peroxide based on immobilization of horseradish peroxidase in a composite membrane of regenerated silk fibroin and poly(vinyl alcohol). Anal Chim Acta, 1996, 329: 97-103

11 Lei $\mathrm{CH}$, Deng JQ. Hydrogen peroxide sensor based on coimmobilized methylene green and horseradish peroxidase in the same montmorillonite-modified bovine serum albumin-glutaraldehyde matrix on a glassy carbon electrode surface. Anal Chem, 1996, 68: 3344-3349

12 Lin H, Cheng HM, Miao XP, Papakonstantinou P, Mihailovič D, Li MX. A novel hydrogen peroxide amperometric sensor based on thionin incorporated onto a $\mathrm{Mo}_{6} \mathrm{~S}_{9-x} \mathrm{I}_{x}$ nanowire modified glassy carbon electrode. Electroanalysis, 2009, 21: 2602-2606

13 Patolsky F, Lichtenstein A, Kotler M, Willner I. Electronic transduction of polymerase or reverse transcriptase induced replication processes 
on surfaces: highly sensitive and specific detection of viral genomes. Angew Chem Int Ed, 2001, 40: 2261-2265

14 Patolsky F, Weizmann Y, Willner I. Redox-active nucleic-acid replica for the amplified bioelectrocatalytic detection of viral DNA. $J$ Am Chem Soc, 2002, 124: 770-772

15 Liu SF, Li YF, Li JR, Jiang L. Enhancement of DNA immobilization and hybridization on gold electrode modified by nanogold agg regates. Biosens Bioelectron, 2005, 21: 789-795

16 Li F, Tang CF, Liu SF, Ma GR. Development of an electrochemical ascorbic acid sensor based on the incorporation of a ferricyanide mediator with a polyelectrolyte-calcium carbonate microsphere. Electrochim Acta, 2010, 55: 838-843

17 Hong YL, Hu NF. Comparative bioelectrochemical study of core-shell nanocluster films with ordinary layer-by-layer films containing heme proteins and $\mathrm{CaCO}_{3}$ nanoparticles. J Phys Chem B, 2005, 109: 10464-10473

18 Abdollah Salimi A, Noorbakhsh A, Mamkhezri H, Ghavamia R. Electrocatalytic reduction of $\mathrm{H}_{2} \mathrm{O}_{2}$ and oxygen on the surface of Thionine incorporated onto MWCNTs modified glassy carbon electrode: application to glucose detection. Electroanalysis, 2007, 19: 1100-1108

19 Ruan CM, Yang F, Chen XH, Deng JQ. Thionine covalently tethered to multilayer horseradish peroxidase in a self-assembled monolayer as an electron-transfer mediator. Anal Chem, 1998, 70: 1721-1725

20 Deng L, Wang YZ, Shang L, Wen D, Wang F, Dong SJ. A sensitive NADH and glucose biosensor tuned by visible light based on thionine bridged carbon nanotubes and gold nanoparticles multilayer. Biosens Bioelectron, 2004, 24: 951-957

21 Xu JJ, Zhou DM, Chen HY. A reagentless hydrogenperoxide biosensor based on the coimmobilization of thionine and horseradish peroxidase by their cross-linking with glutaraldehyde on glassy carbon electrode. Electroanalysis, 1998, 10: 713-716

22 Shi AW, Qu FL, Yang MH, Shen GL, Yu RQ. Amperometric $\mathrm{H}_{2} \mathrm{O}_{2}$ biosensor based on polythionine nanowire/HRP/nano-Au-modified glassy carbon electrode. Sens Actuators B: Chem, 2008, 129: 779-783

23 Shi YT, Yuan R, Chai YQ, Tang MY, He XL. Amplification of antigen-antibody interactions via back-filling of HRP on the layer-by-layer self-assembling of thionine and gold nanoparticles films on titania nanoparticles/gold nanoparticles-coated Au electrode. $J$ Electroanal Chem, 2007, 604: 9-16

24 Li QW, Zhang J, Yan H, He MS, Liu ZF. Thionine-mediated chemistry of carbon nanotubes. Carbon, 2004, 42: 287-291

25 Wang B, Dong S. Sol-gel-derived amperometric biosensor for hydrogen peroxide based on methylene green incorporated in Nafion film. Talanta, 2000, 51: 565-572

26 Shahrokhian S, Zare-Mehrjardi HR. Application of thionine-Nafion supported on multi-walled carbon nanotube for preparation of a modified electrode in simultaneous voltammetric detection of dopamine and ascorbic acid. Electrochim Acta, 2007, 52(22): 6310-6317

27 Ramirez Molina C, Boujtita M, El Murr N. A carbon paste electrode modified by entrapped toluidine blue-O for amperometric determination of L-lactate. Anal Chim Acta, 1999, 401: 155-162

28 Xu XY, Feng Y, Li JJ, Li F, Yu HJ. A novel protocol for covalent immobilization of thionine on glassy carbon electrode and its application in hydrogen peroxide biosensor. Biosens Bioelectron, 2010, 25: 2324-2328

29 Zhang MG, Gorski W. Electrochemical sensing platform based on the carbon nanotubes/redox mediators-biopolymer system. $J$ Am Chem Soc, 2005, 127: 2058-2059

30 Xu Y, Jiang Y, Yang L, He PG, Fang YZ. Direct electrochemical detection of oligonucleotide hybridization on poly(thionine) film. Chin J Chem, 2005, 23: 1665-1670

31 Salimi A, Noorbakhsh A, Soltanian S. Electroless deposition of thionin onto glassy carbon electrode modified with single wall and multiwall carbon nanotubes: improvement of the electrochemical reversibility and stability. Electroanalysis, 2006, 18: 703-711

32 Cai WY, Xu Q, Zhao XN, Zhu JJ, Chen HY. Porous gold-nanoparticle- $\mathrm{CaCO}_{3}$ hybrid material: preparation, characterization, and application for horseradish peroxidase assembly and direct electrochemistry. Chem Mater, 2006, 18: 279-284

33 Volodkin DV, Larionova NI, Sukhorukov GB. Protein encapsulation via porous $\mathrm{CaCO}_{3}$ microparticles templating. Biomacromolecules, 2004, 5: 1962-1972

34 Jiao LS, Niu L, Shen J, You TY, Dong SJ, Ivaska A. Simple azo derivatization on 4-aminothiophenol/Au monolayer. Electrochem Commun, 2005, 7: 219-222

35 Ferapontova EE. Direct peroxidase bioelectrocatalysis on a variety of electrode materials. Electroanalysis, 2004, 16: 1101-1112

36 Laviron E. Adsorption, autoinhibition and autocatalysis in polarography and in linear potential sweep voltammetry. J Electroanal Chem, 1974, 52: 355-393

37 Shobha Jeykumari DR, Ramaprabhu S, Sriman Narayanan S. A thionine functionalized multiwalled carbon nanotubemodified electrode for the determination of hydrogen peroxide. Carbon, 2007, 45: 1340-1353

38 Shan D, Wang SX, Xue HG, Cosnier S. Direct electrochemistry and electrocatalysis of hemoglobin entrapped in composite matrix based on 
chitosan and $\mathrm{CaCO}_{3}$ nanoparticles. Electrochem Commun, 2007, 9: 529-534

39 Li F, Chen W, Tang CF, Zhang SS. Development of hydrogen peroxide biosensor based on in situ covalent immobilization of horseradish peroxidase by one-pot polysaccharide-incorporated sol-gel process. Talanta, 2009, 77: 1304-1308

40 Kamin RA, Wilson GS. Rotating ring-disk enzyme electrode for biocatalysis kinetic studies and characterization of the immobilized enzyme layer. Anal Chem, 1980, 52: 1198-1205

\title{
A novel strategy for immobilization of thionine based on calcium carbonate-gold nanoparticles inorganic hybrid composite and its application in hydrogen peroxide sensor
}

\author{
LI Feng $^{1}$, FENG Yan $^{1}$, LI JingJing ${ }^{1} \&$ GUO Jie ${ }^{2}$
}

1 Key Laboratory of Eco-Chemical Engineering, Ministry of Education; College of Chemistry and Molecular Engineering, Qingdao University of Science and Technology, Qingdao 266042, China

2 Department of Radiotherapy, Second Hospital, Jilin University, Changchun 130025, China

\begin{abstract}
A novel strategy for efficient immobilization of electroactive Thionine (Th) on gold (Au) electrode surface based on calcium carbonate-gold nanoparticles $\left(\mathrm{CaCO}_{3}-\mathrm{AuNPs}\right)$ inorganic hybrid composite was proposed. It was conducted by the strong electrostatic interaction between positively charged $\mathrm{Th}$ and negatively charged $\mathrm{CaCO}_{3}$-AuNPs composite. The hybrid composite was obtained by the adsorption of AuNPs onto the surface of $\mathrm{CaCO}_{3}$ microspheres through electrostatic interaction. Due to the microporous architecture, large surface area, and good biocompatibility of $\mathrm{CaCO}_{3}$-AuNPs composite, the amount and stability of the immobilized Th were highly strengthened. The application of the resulting Th modified electrode in hydrogen peroxide $\left(\mathrm{H}_{2} \mathrm{O}_{2}\right)$ sensor was also investigated and it exhibited rapid response to $\mathrm{H}_{2} \mathrm{O}_{2}$ within $3 \mathrm{~s}$. The linear calibration ranged from $8.00 \times 10^{-7}$ to 1.06 $\times 10^{-3} \mathrm{~mol} / \mathrm{L}$ with a detection limit of $2.00 \times 10^{-7} \mathrm{~mol} / \mathrm{L}$.
\end{abstract}

Keywords: thionine, $\mathrm{CaCO}_{3}-\mathrm{AuNPs}$, sensor, electrostatic interaction 